\title{
ARTICLE \\ Alterations in interhemispheric gamma-band connectivity are related to the emergence of auditory verbal hallucinations in healthy subjects during NMDA-receptor blockade
}

\author{
Stephanie Thiebes ${ }^{1}$, Saskia Steinmann ${ }^{1}$, Stjepan Curic ${ }^{1}$, Nenad Polomac ${ }^{1}$, Christina Andreou ${ }^{1,2}$, Iris-Carola Eichler ${ }^{3}$, Lars Eichler ${ }^{3}$, \\ Christian Zöllner ${ }^{3}$, Jürgen Gallinat ${ }^{4}$, Gregor Leicht ${ }^{1}$ and Christoph Mulert ${ }^{1}$
}

\begin{abstract}
Auditory verbal hallucinations (AVH) are a common positive symptom of schizophrenia. Excitatory-to-inhibitory (E/I) imbalance related to disturbed $\mathrm{N}$-methyl-D-aspartate receptor (NMDAR) functioning has been suggested as a possible mechanism underlying altered connectivity and AVH in schizophrenia. The current study examined the effects of ketamine, a NMDAR antagonist, on glutamate-related mechanisms underlying interhemispheric gamma-band connectivity, conscious auditory perception during dichotic listening (DL), and the emergence of auditory verbal distortions and hallucinations (AVD/AVH) in healthy volunteers. In a single-blind, pseudo-randomized, placebo-controlled crossover design, nineteen male, right-handed volunteers were measured using 64 channel electroencephalography (EEG). Psychopathology was assessed with the PANSS interview and the 5D-ASC questionnaire, including a subscale to detect auditory alterations with regard to AVD/AVH (AUA-AVD/AVH). Interhemispheric connectivity analysis was performed using eLORETA source estimation and lagged phase synchronization (LPS) in the gamma-band range $(30-100 \mathrm{~Hz})$. Ketamine induced positive symptoms such as hallucinations in a subgroup of healthy subjects. In addition, interhemispheric gamma-band connectivity was found to be altered under ketamine compared to placebo, and subjects with AUAAVD/AVH under ketamine showed significantly higher interhemispheric gamma-band connectivity than subjects without AUAAVD/AVH. These findings demonstrate a relationship between NMDAR functioning, interhemispheric connectivity in the gammaband frequency range between bilateral auditory cortices and the emergence of AVD/AVH in healthy subjects. The result is in accordance with the interhemispheric miscommunication hypothesis of $\mathrm{AVH}$ and argues for a possible role of glutamate in AVH in schizophrenia.
\end{abstract}

Neuropsychopharmacology (2018) 43:1608-1615; https://doi.org/10.1038/s41386-018-0014-z

\section{INTRODUCTION}

Auditory verbal hallucinations (AVH) - vocal perceptions in the absence of an actual auditory stimulus [1] -are one of the most common positive symptoms in schizophrenia. Recent findings from brain imaging have associated $\mathrm{AVH}$ with alterations in a brain network related to speech and language processing [2]. Alterations in connectivity patterns have been suggested to relate to current concepts of the emergence of $\mathrm{AVH}$, such as the interhemispheric miscommunication hypothesis [3]. In this context, alterations in high-frequency $(30-100 \mathrm{~Hz})$ gamma-band oscillations (GBO) are consistently discussed [4], given their role in synchronizing neural activity within and between distributed brain structures. Although it is known that GBO have a specific role for perception processes in general $[5,6]$, and that alterations of GBO are a consistent finding in schizophrenia [4], our understanding of their role for $A V H$ is still far from sufficient.

According to the interhemispheric miscommunication hypothesis, altered connectivity between bilateral auditory areas contributes to the underlying neural correlate of AVH. The hypothesis is based on multimodal evidence including diffusion tensor imaging (DTI), functional magnetic resonance imaging (fMRI) and electroencephalography (EEG) findings. DTI studies have shown that alterations of interhemispheric auditory pathways (IAP) are related to the appearance of AVH [7-9]. In addition, disturbed functional connectivity in the IAP have been found to be related to AVH using fMRI [10]. Moreover, a significant positive correlation was found between AVH severity and increased interhemispheric gamma-band coupling between bilateral auditory cortices [11]. Recently, Steinmann et al. [12] showed that AVH in schizophrenia are related to stronger interhemispheric gammaband connectivity (lagged phase synchronization) during conscious perception of the left ear (LE) syllable in a dichotic listening (DL) task.

In healthy subjects, interhemispheric communication is relevant to conscious auditory perception in a DL task. Although both ears are connected anatomically via ipsilateral and contralateral pathways with the auditory cortices, ipsilateral pathways are suggested to be blocked during DL [13] leading to supremacy of contralateral pathways. Accordingly, auditory speech stimuli presented to the LE initially access the right hemisphere, but require further transfer

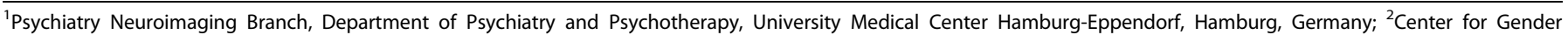

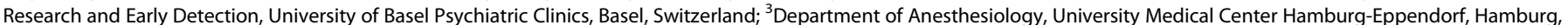
Germany and ${ }^{4}$ Department of Psychiatry and Psychotherapy, University Medical Center Hamburg-Eppendorf, Hamburg, Germany

Correspondence: Christoph Mulert (c.mulert@uke.uni-hamburg.de)

Received: 4 July 2017 Revised: 19 December 2017 Accepted: 5 January 2018

Published online: 5 February 2018 
A

\section{LE Percept}

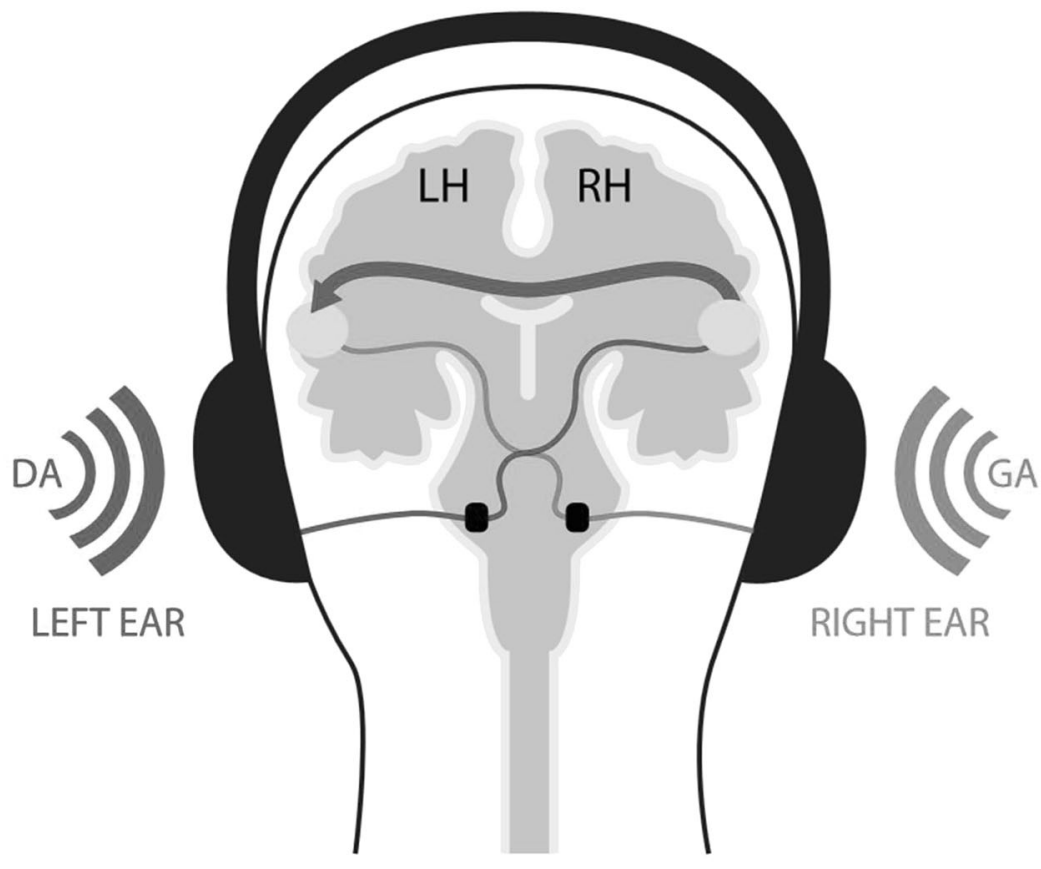

B
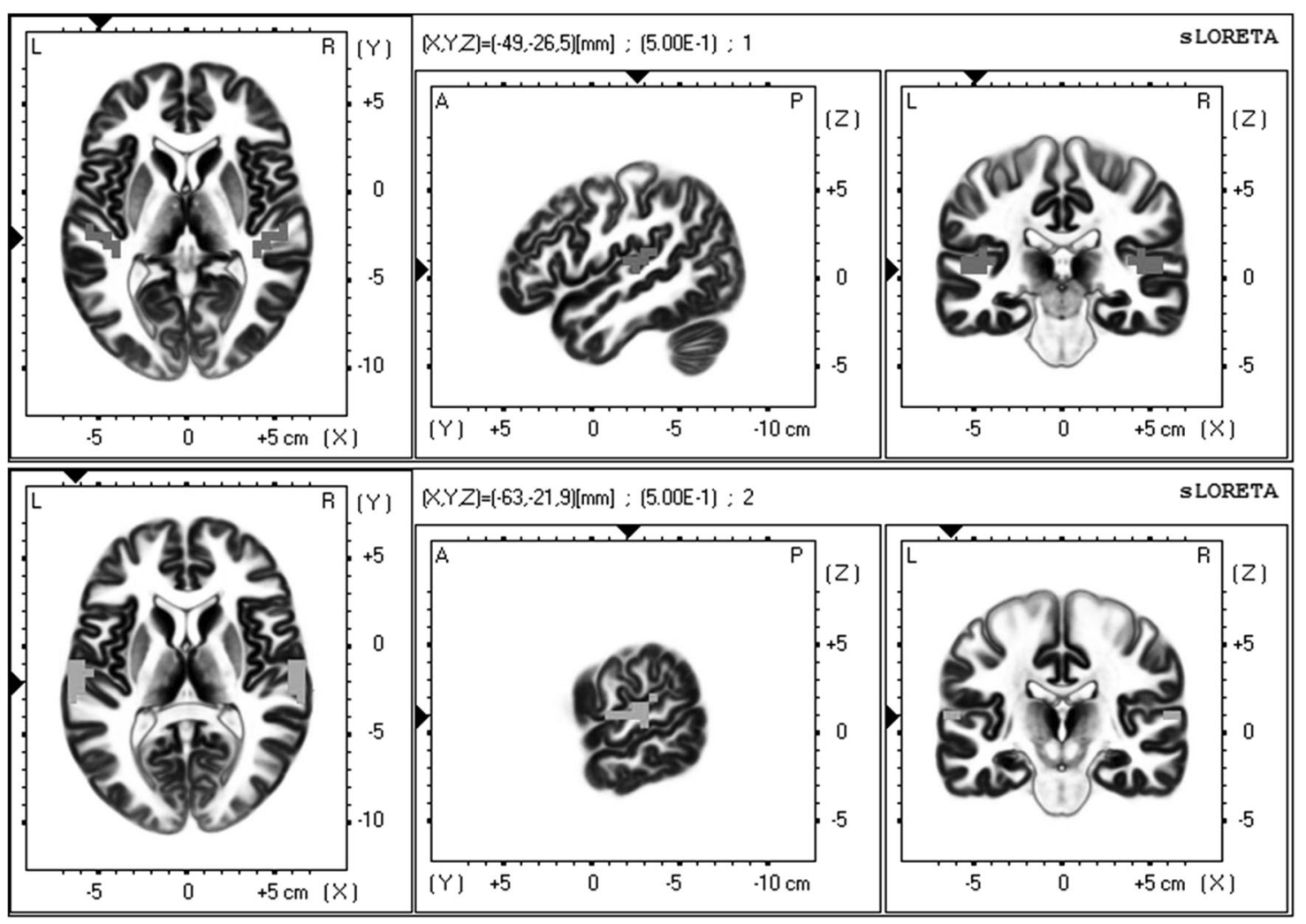

across the corpus callosum (CC) to be efficiently processed in the speech-dominant left hemisphere [14] (Fig. 1a). This interhemispheric auditory transfer has been shown to be mediated by synchronized GBO [15], and further, to be particularly increased in schizophrenia patients hearing voices [12].
Gamma rhythms are dependent on the activity of parvalbumincontaining (PV) gamma-aminobutyric acid-(GABA-)ergic cortical inhibitory interneurons, which are subject to glutamate $N$-methylD-Aspartate receptor (NMDAR) modulation from excitatory pyramidal cells [16]. As disturbed gamma rhythms have been 
Fig. 1 a Schematic illustration of the dichotic listening (DL) procedure, demonstrating the requirement of interhemispheric transfer when participants consciously perceive the auditory stimulus presented to the left ear (LE). Two different syllables are presented simultaneously: one to the right ear (GA) and one to the left ear (DA). Although both ears are connected anatomically via ipsilateral and contralateral pathways with the auditory cortices, ipsilateral pathways are suggested to be blocked during dichotic listening. The blue line indicates the contralateral pathway connecting the stimulus presented to the right ear (RE) directly with the speech-dominant left hemisphere. In contrast, the syllable arriving from the LE to the right hemisphere requires interhemispheric transfer via the corpus callosum (CC) to be efficiently processed (red line). Yellow: right and left auditory cortices, $\mathrm{LH}$ : left hemisphere, $\mathrm{RH}$ : right hemisphere. $\mathbf{b}$ Regions-of-interests (ROIs) within the left and right primary auditory cortices (PACs, BA41, red) and the left and right secondary auditory cortices (SACs, BA42, green). BA41-ROI covered a region extended from $x$ : 35 to 55 and -35 to $-55, y:-15$ to $-40, z$ : 5 to 15 and included all voxels of BA41. BA42-ROI covered a region extended from $x: 55$ to 70 and -55 to $-65, y:-10$ to $-35, z: 5$ to 20 and included all voxels of BA42

reported in schizophrenia consistently $[17,18]$, there has been growing interest in the role of inhibitory interneurons and their neural circuits that are responsible for maintaining a precise excitatory-to-inhibitory (E/I) balance [19]. Recent reviews have suggested that an impaired $\mathrm{E} / \mathrm{I}$ balance is crucially involved in schizophrenia [20-22] and in the emergence of AVH [23]. Moreover, phase synchronization of oscillatory activity depends on E/I balance [23], and has been shown to be increased in the gamma-band between bilateral auditory cortices in schizophrenia patients with AVH [11]. Furthermore, NMDAR antagonists such as ketamine induce an E/I imbalance [24], and are known to alter GBO [25] and to elicit schizophrenia-like symptoms, including AVH [26-28], depending on the ketamine dose, in healthy volunteers. Whereas low doses $(0.003 \mathrm{mg} / \mathrm{kg} / \mathrm{min})$ of ketamine already produce milder symptoms such as reduced vigilance, only high doses of ketamine (at least $0.005 \mathrm{mg} / \mathrm{kg} / \mathrm{min}$ ) are able to elicit auditory alterations [29]. This could be interpreted as evidence for a threshold dose to induce hallucinatory activity which is capable of disturbing the auditory perceptual system.

Given that increased GBO during LE percepts in a DL task are associated with AVH in schizophrenia patients, and that ketamine induces schizophrenia-like symptoms and influences GBO, we hypothesized that high-dose ketamine administered to healthy volunteers (1) elicits schizophrenia-like symptoms including $\mathrm{AVH}$, (2) alters interhemispheric gamma-band connectivity during LE percepts, and that (3) enhanced interhemispheric gamma-band connectivity is particularly related to the occurrence of ketamineinduced auditory verbal distortions and hallucinations (AVD/AVH).

\section{MATERIALS AND METHODS}

\section{Participants}

Participants were recruited from the community through advertisement and word-of-mouth. Overall, 25 male German native speakers were enrolled in this study, who were either medical students or medical staff of the University Medical Center Hamburg. Inclusion criteria were male gender, age between 18 and 40 years, right-handedness, normal or corrected visual acuity, German at native speaker level, and normal hearing. Two of the volunteers dropped out due to adverse effects (dissociative effect/ headache) during the ketamine session and were not able to continue with testing. Two further participants withdrew consent shortly before the first session. Two further participants did not fulfill criteria for further EEG analysis, i.e., $<30$ segments remained in one of the conditions (right ear (RE) or LE reports) after EEG preprocessing. For the following data analysis 19 subjects with a mean age of 25 years $(S D=2.32$, range $=20-29$ ) were included. The sample of the study is part of a larger project, of which some results have been published already [30].

All subjects were screened with pure tone audiometry for the frequencies of 120, 250,500,1000, 2000,4000, and $8000 \mathrm{~Hz}$ (Esser Home Audiometer 2.0) in order to ensure normal hearing in both ears. Participants with an auditory threshold higher than $20 \mathrm{~dB}$, or an interaural difference larger than $15 \mathrm{~dB}$ in any of the frequencies were excluded from the study. All volunteers were right-handed, assessed with the empirically validated Edinburgh Handedness
Inventory [31] (mean $=84.55, \mathrm{SD}=16.19$, range $=40-100)$. Exclusion criteria were the use of illicit drugs, alcohol consumption of more than seven units per week, current medication, a positive family history of schizophrenia or bipolar disorder, acute or past psychiatric disorders - tested with the Mini International Neuropsychiatric Interview [32] and the Schizotypal Personality Questionnaire (SPQ) [33] —cardiovascular diseases, hyperthyroidism and hypothyroidism, and a ketamine intolerance. All participants had normal IQ as tested with a vocabulary test [34] (mean = $112.00, S D=5.58$, range $=99-122$ ). The general procedure was approved by the ethical committee of Medical Association Hamburg. After participants received a complete description of the experimental procedure, written informed consent was obtained according to the Declaration of Helsinki.

\section{Study design}

We used a single-blind, randomized, placebo-controlled crossover study design. During the ketamine session a subanesthetic dose of $S$-ketamine Hydrochloride (Ketanest ${ }^{\circledR} \mathrm{S}$ - Pfizer) was administered intravenously in $0.9 \%$ sodium chloride $(\mathrm{NaCl})$ solution using a syringe pump (Perfusor ${ }^{\circledR}$ Space) for $75 \mathrm{~min}$. The ketamine infusion was started with an initial bolus of $10 \mathrm{mg}$ over $5 \mathrm{~min}$ followed by a maintenance infusion of $0.006 \mathrm{mg} / \mathrm{kg} / \mathrm{min}$. As ketamine plasma levels slowly increase with continuous infusion, the dosage was reduced by $10 \%$ every $10 \mathrm{~min}$. Placebo was administered analogously as $0.9 \% \mathrm{NaCl}$ infusion. Heart rate, blood pressure and oxygen saturation were continuously monitored during both sessions by a board certified anesthesiologist. After both sessions the subjects stayed under constant supervision for one hour, until all drug effects had worn off.

\section{Psychometric assessment}

Psychiatric symptomatology was assessed using the Positive and Negative Syndrome Scale (PANSS) [35], including one item detecting hallucinations (P3) in any sensory modality (visual, olfactory, somatic and auditory). Prior to the first intervention (baseline) and after both drug challenges (placebo/ketamine) psychopathological evaluation was conducted by an experienced psychiatrist. PANSS scores were evaluated using the five-factor model by van der Gaag et al. [36].

To assess the subjective effects of ketamine and to detect auditory hallucinations specifically, the Altered State of Consciousness (5D-ASC) questionnaire [37], a visual analog scale, was used after both drug challenges (ketamine/placebo). This self-rating questionnaire consists of 94 items/statements assessing five key dimensions of altered states of consciousness (ASC): dread of ego dissolution (DED), auditory alterations (AUA), oceanic boundlessness $(\mathrm{OBN})$, vigilance reduction (VIR), and visionary restructuralization (VRS). The scale was previously shown to be sensitive to the psychological effects of ketamine [27]. With respect to AVH in schizophrenia, we derived a more specific AUA subscore that included only AUA related to auditory verbal distortions (AVD) and AVH (AUA-AVD/AVH) in contrast to non-verbal auditory distortions and hallucinations. The AUA score includes 16 items of the 5D-ASC (items: 4, 5, 11, 13, 19, 25, 30, 48, 49, 55, 65, 66, 74, 76, 92, and 93), the AUA-AVD/AVH scores includes 6 items $(4,13,19,25,30$, and 65$)$. 
A

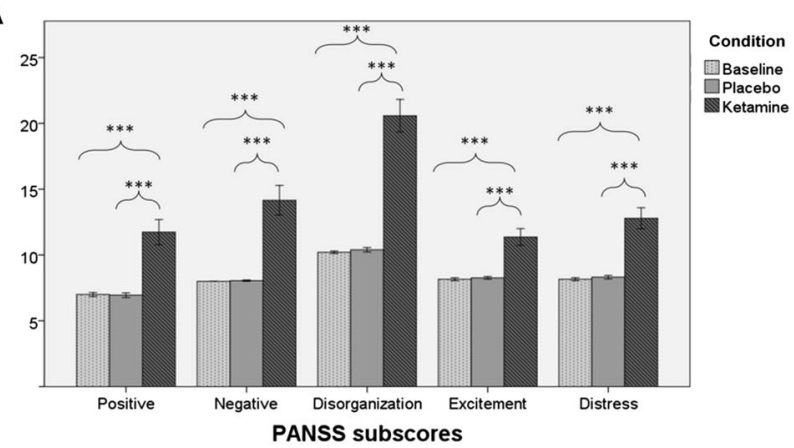

B
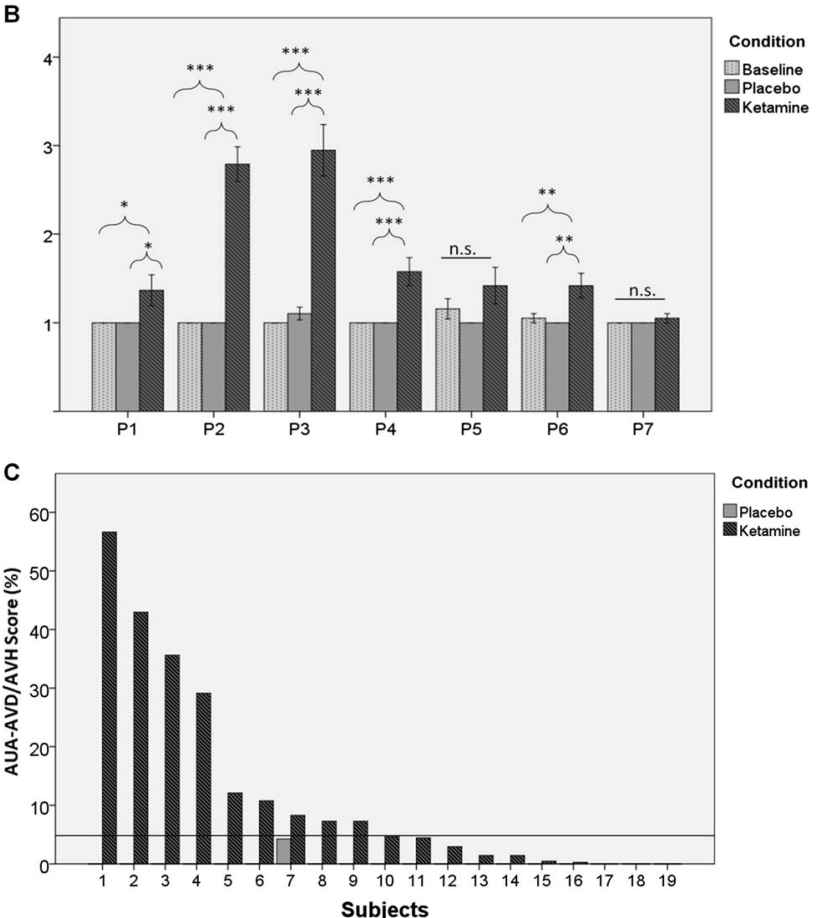

Fig. 2 Bar charts of mean values of the five PANSS subscores (a) and the positive items (b) of the PANSS with error bars representing \pm 1 standard error. Bar charts of the subscore auditory alterations in the form of auditory verbal distortions and hallucinations (AUA-AVD/ AVH) of the 5D-ASC (in \%) (c) for each subject in descending order under ketamine administration compared to placebo administration. The black, horizontal line indicates where the median split was conducted. $\mathrm{P} 1=$ delusion; $\mathrm{P} 2=$ conceptual disorganization; $\mathrm{P} 3=$ hallucinations; $\mathrm{P} 4=$ excitement; $\mathrm{P} 5=$ grandiosity; $\mathrm{P} 6=$ suspiciousness/ persecution; P7 = hostility. ${ }^{*} p<0.05 ;{ }^{* *} p<0.01 ;{ }^{* * *} p<0.001 ; n=19$

Items were only included in the AUA-AVD/AVH score, if their corresponding statements contained the words "voice/s" "to speak" and/or "words". For further details see Table S1. The participants were carefully assigned according to the current history of AVD and/ or $\mathrm{AVH}$ under ketamine using the AUA-AVD/AVH score into two groups (AVD/AVH vs. non-AVD/AVH). This results in a non-AVD/AVH group of 10 participants who reported AUA-AVD/AVH on a level comparable to placebo, and in an AVD/AVH group of nine participants. Scores of the single items of the AUA-AVD/AVH score of both groups and placebo are shown in Figure S1.

Stimuli

The Bergen dichotic listening (DL) paradigm consists of six consonant-vowel (CV) syllables /ba/, /da/, /ga/, /pa/, /ta/ and / ka/ , which were paired and presented simultaneously (Fig. 1a). All syllables were spoken by a German male speaker with constant intensity and intonation and with duration of $400-500 \mathrm{~ms}$.
Participants were instructed to report after each trial the syllable which was perceived. The response was given via button press with the right dominant hand. On the behavioral level a laterality index (LI), error rates and reaction times were evaluated. For detailed description see Steinmann et al. [15].

Recording and pre-processing

EEG recording took place in a sound-attenuated and electrically shielded cabin. Continuous EEG activity was recorded using Ag/ $\mathrm{AgCl}$ electrodes mounted in a 64 channel actiCAP system (recording apparatus: Brain Products $\mathrm{GmbH}$, Munich, Germany). EEG pre-processing was carried out using the Brain Vision Analyzer software version 2.0 (Brain Products, Munich, Germany). For detailed description see Steinmann et al. [15] and Supplementary Materials and Methods. After EEG pre-processing, trials were divided according to RE or LE percept. For all segments, $200 \mathrm{~ms}$ pre-stimulus to $1848 \mathrm{~ms}$ post-stimulus periods were created. The number of EEG segments used for analysis was balanced between LE and RE reports for every single subject resulting in a mean number of 76.95 (SD =20.09) segments for each of the conditions. For the analyses of the N100 potential, auditory-evoked gammaband response (aeGBR) and baseline gamma power see Supplementary Materials and Methods.

Functional connectivity analysis

All further analyses were executed with the LORETA KEY software package as provided by Roberto Pascual-Marqui (The KEY Institute for Brain-Mind Research University Hospital Psychiatry, Zurich) at www.uzh.ch/keyinst/LORETA.html. The eLORETA method is a discrete, three-dimensional (3D) distributed, linear, and weighted minimum norm inverse solution that has the property of exact localization to test point sources $[38,39]$.

For analysis of functional connectivity between auditory cortices the lagged phase synchronization (LPS) approach was applied. This method measures the similarity between signals in the frequency domain based on normalized (unit module) Fourier transforms, and thus, is related to nonlinear functional connectivity [40,41]. For further details see Supplementary Materials and Methods.

In this study LPS was assessed within two a priori defined regions-of-interests (ROIs), which have been identified as essential nodes in the AVH network [15]: right and left primary (PACs/BA41) and secondary (SACs/BA42) auditory cortices (Fig. 1b). The ROls were defined using the anatomical definitions based on the Talairach Daemon (http://www.talairach.org//). As a previous study by Steinmann et al. [15] did not find LPS differences in any other frequencies than gamma, we focused on the whole gamma-band range $(30-100 \mathrm{~Hz})$. LPS across all the voxels included in the ROls was based on the average of all LPS values, which were calculated for the connectivity between every voxel of each ROI. For all single trials, time-varying frequency analysis was done using a short time Fourier transform (sliding Bartlett-Hann window function) with a window width of $100 \mathrm{~ms}$. LPS was calculated using cross spectra derived from that transformation. Results were extracted every $100 \mathrm{~ms}$ in a time frame from 0 to $900 \mathrm{~ms}$ after stimulus presentation. In order to ensure that no baseline correction is required, LPS results were additionally extracted in the prestimulus baseline from 200 to $0 \mathrm{~ms}$.

Statistics

All data analyses were performed using SPSS version 21. Behavioral data of DL task performance (LI, reaction times and error rates) between placebo and ketamine condition were conducted using paired-sample t-tests. Comparisons of 5D-ASC scores between placebo and ketamine condition, and further, between the AVD/AVH and non-AVD/AVH group, were conducted using paired-sample $t$-tests. To test the reliability of the AUA-AVD/ $\mathrm{AVH}$ score, we analyzed internal consistency measured with Cronbach's $a$. PANSS scores were analyzed by means of one-way 


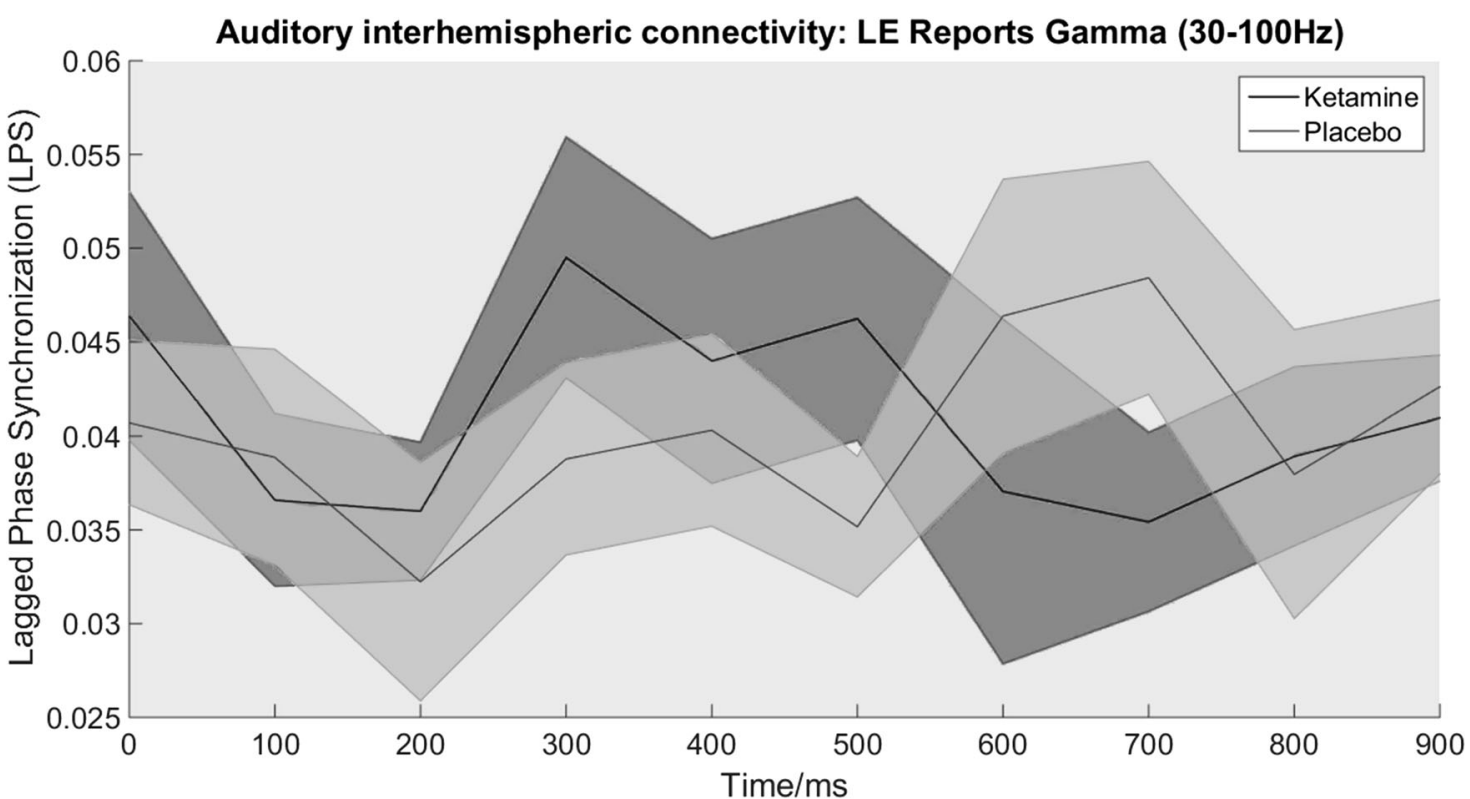

Fig. 3 Time course of interhemispheric auditory connectivity in the gamma-band $(30-100 \mathrm{~Hz})$ between bilateral auditory cortices (BA41 and BA42) during left ear (LE) reports for ketamine (red line) and placebo (blue line) ( $n=19)$. Syllable presentation starts at 0 ms and ends around $500 \mathrm{~ms}$. The quality of gamma-band connectivity was found to be significantly altered under ketamine administration in contrast to placebo. Shaded error bars: \pm 1 standard error

repeated measure analysis of variance (RM-ANOVA) with condition (baseline, placebo, and ketamine) as within-subject factor and post-hoc $t$-tests. Bonferroni correction was applied for multiple comparisons. Spearman's correlation coefficient was used in order to investigate the relation between gamma connectivity (mean LPS difference between ketamine and placebo for LE reports) and the AUA-AVD/AVH score. The effects of substance, condition, ROI and time on gamma connectivity were assessed with a linear mixed-effects model. Linear mixed models (LMM) have several advantages over traditional repeated-measures designs, as they can accommodate departures from the assumptions of homogeneity of regression slopes and independence, and thus, are better suited to model interindividual variability [42]. This model has also been successfully implemented by researchers to analyze EEG data $[43,44]$. In this study, the model included Substance (2 levels: placebo, ketamine), Condition (2 levels: LE and RE reports), ROI (2 levels: BA41, BA42) and Time(10 levels: from 0 to $900 \mathrm{~ms}$ in $100 \mathrm{~ms}$ steps) as the fixed-effects factors, subject as a randomeffects factor, and Gamma Connectivity as the dependent variable. In follow-up analyses Group (2 levels: AVD/AVH and non-AVD/AVH subjects) was included in order to differentiate more precisely between subjects with and without AVD/AVH under ketamine administration. LMM analysis for the pre-stimulus (from -200 to 0 $\mathrm{ms}$ ) interval was conducted separately. In order to test the specificity of the results we repeated the follow-up analysis for all other subscores of the 5D-ASC and the PANSS hallucination item P3. In all analyses, the significance level was set to $a=0.05$.

\section{RESULTS}

Ketamine-induced psychopathological symptoms

The RM-ANOVA revealed significant main effects of condition (baseline, placebo and ketamine) for PANSS score total $\left(F_{(2,56)}=\right.$ 79.25, $p<0.001)$, positive $\left(F_{(2,56)}=23.47, p<0.001\right)$, negative $\left(F_{(2,56)}=29.59, \quad p<0.001\right]$, disorganization $\left(F_{(2,56)}=66.68, \quad p<\right.$ $0.001)$, excitement $\left(F_{(2,56)}=23.28, p<0.001\right)$, and distress $\left(F_{(2,56)}\right.$ $=30.86, p<0.001$ ) (Fig. 2a). The same was the case for the PANSS positive hallucination item P3 $\left(F_{(2,56)}=40.04, p<0.001\right)$ (Fig. $\left.2 b\right)$. Post-hoc $t$-tests showed that all subscores of the PANSS as well as the positive item P3 (hallucinations) were significantly increased after ketamine administration in comparison to placebo administration and baseline values. There were no significant differences with respect to any PANSS score and positive item between baseline and placebo condition (Fig. 2). Moreover, 5D-ASC scores (Figure S2), including the AUA-AVD/AVH score $(t=3.05 ; p=0.042$ after Bonferroni correction; Fig. 2c), were significantly increased after ketamine administration compared to placebo administration. The subgroups (AVD/AVH, non-AVD/AVH) did not differ in any other $5 \mathrm{D}$-ASC subscore (all $p>0.15$ ). The analysis of internal consistency of the AUA-AVD/AVH score revealed a good level of reliability (Cronbach's $a=0.889$ ).

Dichotic listening task performance

Reaction times were significantly increased under ketamine compared to placebo for LE reports (placebo: mean $=2.63 \mathrm{~s}$; SD $=0.31 \mathrm{~s}$; ketamine: mean $=3.20 \mathrm{~s} ; \mathrm{SD}=0.47 \mathrm{~s} ; t=4.58 ; p<0.001$ ) as well as for RE reports (placebo: mean $=2.54 \mathrm{~s} ; \mathrm{SD}=0.28 \mathrm{~s}$; ketamine: $\operatorname{mean}=3.15 \mathrm{~s} ; \mathrm{SD}=0.42 \mathrm{~s} ; t=5.10 ; p<0.001$ ). There were no significant differences between ketamine and placebo in $\mathrm{LI}$ and error rates $(p>0.179)$. For detailed information see Table S2.

N100, baseline, and evoked gamma power

At sensor level, auditory presentation of CV-syllables elicited the typical N100 component of the auditory-evoked potential for placebo and ketamine (Figure S3). In addition, time-frequency analysis using complex Morlet wavelet transformation showed the typical auditory-evoked gamma-band response (aeGBR) in the frequency range around $40 \mathrm{~Hz}$ after syllable presentation for both conditions (Figure S4). There were no significant difference of the aeGBR power and the N100 potential between ketamine and placebo $(p>0.45)$. Baseline gamma power was significantly increased by ketamine $(F=9.72, p=0.006)$ across all scalp regions (Figure S5). There were no relations between N100, aeGBR or baseline gamma power, and AUA-AVD/AVH.

Functional connectivity by means of lagged phase synchronization (LPS)

There were no significant differences in baseline LPS (-200 to 0 $\mathrm{ms}$ ) between ketamine and placebo as well as between the AVD/ $\mathrm{AVH}$ and non-AVD/AVH group, and no significant effect or 
A

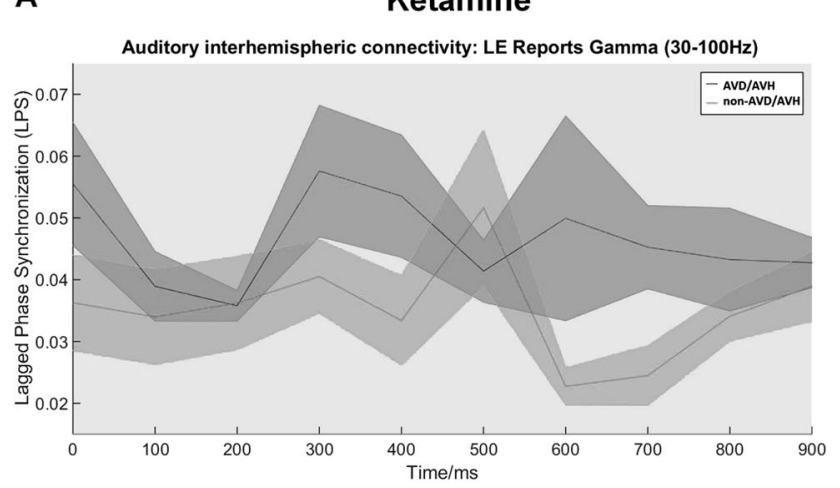

C

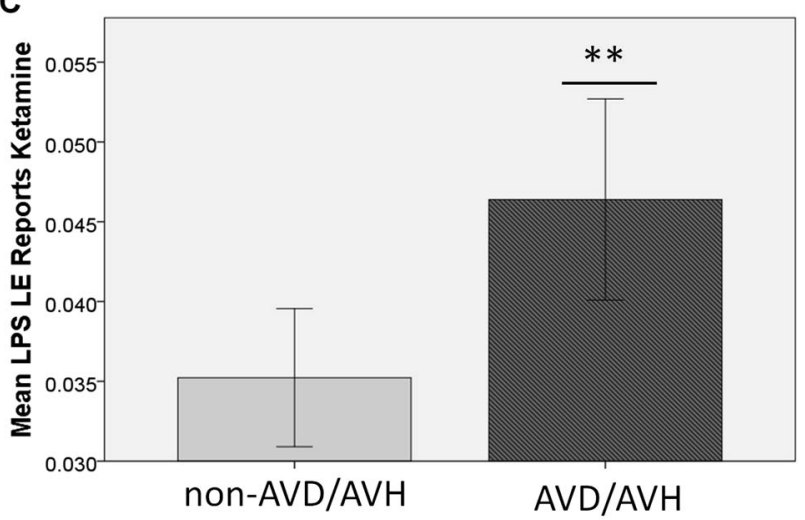

Group
B

\section{Placebo}

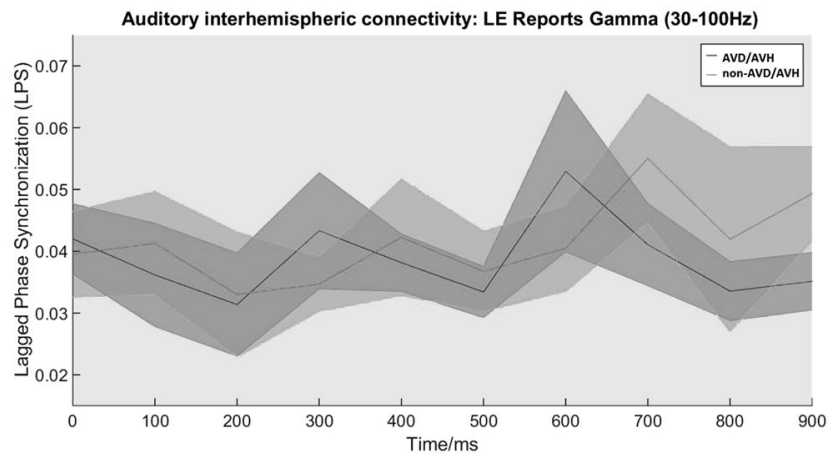

D

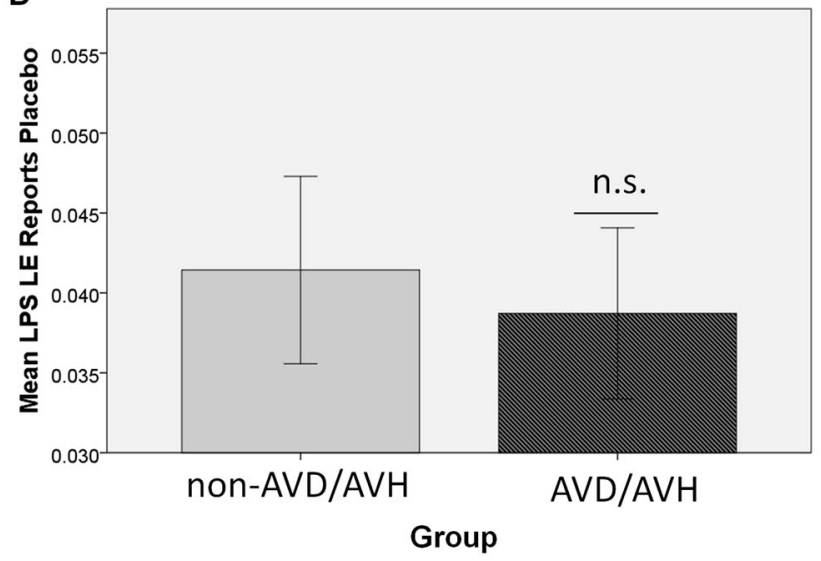

Fig. 4 Time course of interhemispheric auditory connectivity in the gamma-band $(30-100 \mathrm{~Hz})$ between bilateral auditory cortices (BA41 and BA42) during left ear (LE) reports for AVD/AVH ( $n=9$; red line) and non-AVD/AVH ( $n=10$; cyan line) subjects under ketamine (a) and under placebo (b) administration. Syllable presentation starts at $0 \mathrm{~ms}$ and ends around $500 \mathrm{~ms}$. Shaded error bars: \pm 1 standard error. Gamma-band connectivity was significantly increased in the AVD/AVH group compared to the non-AVD/AVH group over time under ketamine administration: bar charts of mean lagged phase synchronization (LPS) during LE reports under ketamine (c) and under placebo (d) administration for AVD/AVH $(n=9)$ and non-AVD/AVH $\left(n=10\right.$; with error bars representing \pm 1 standard error). ${ }^{* *} p<0.01$; n.s. $=$ not significant

interaction of $\mathrm{ROI}$ in any of the analyses. The three-way Substance $\times$ Time $\times$ Condition interaction had a significant effect on Gamma Connectivity $\left(F_{(9,902.478)}=2.027, p=0.034\right)$.

In order to further clarify this interaction, follow-up LMMs were conducted (see also Supplementary Results) separately for LE and RE reports, with Substance, Time and $R O I$ as fixed factors and Gamma Connectivity as dependent variable. This analysis revealed that the interaction of Time $\times$ Substance had a significant effect on Gamma Connectivity for LE reports $\left(F_{(9,578.560)}=2.605, p=0.006\right]$ (Fig. 3) but not for RE reports $\left(F_{(9,608.998)}=0.963, p>0.470\right)$, indicating an altered time course of gamma-band connectivity during LE reports under ketamine compared to placebo. Therefore, all subsequent analyses focused on gamma connectivity during LE reports. Subsequent post-hoc pair wise comparison of every time point between placebo and ketamine during LE reports revealed stronger interhemispheric gamma-band connectivity at statistical trend level at $300 \mathrm{~ms}(t=1.998, p=0.061)$ and $500 \mathrm{~ms}$ $(t=1.916, p=0.071)$ post-stimulus for ketamine.

Functional connectivity differences during LE reports between AVD/AVH and non-AVD/AVH subjects were investigated with a LMM including Substance, Time and $\mathrm{ROI}$ as within-subjects factors, and Group (AVD/AVH vs. non-AVD/AVH) as between-subject factor. This analysis revealed a significant interaction of Substance $x$ Group $\left(F_{(1,207.690)}=8.151, p=0.005\right)$. Follow-up LMM analyses conducted for each Substance separately showed a significant main effect of group for ketamine $\left(F_{(1,82.609)}=7.215, p=0.009\right)$ but not for placebo $\left(F_{(1,79.364)}=1.372, p>0.245\right)$ (Fig. 4), indicating higher gamma-band connectivity for AVD/AVH compared to non-
AVD/AVH under ketamine during LE reports. This analysis did not reveal significance for any other $5 \mathrm{D}$-ASC subscore and neither for P3 $(p>0.154)$, indicating the specificity of the AUA-AVD/AVH score. A positive correlation between the AUA-AVD/AVH score and the mean LPS difference score $(r=0.311, p=0.194)$ did not reveal significance, although the correlation coefficient already indicates a medium effect size. There was no significant correlation between baseline gamma power and interhemispheric gammaband connectivity under ketamine.

\section{DISCUSSION}

In accordance with our hypothesis, the main findings were that ketamine (1) elicited schizophrenia-like symptoms including $\mathrm{AVH}$, (2) altered interhemispheric gamma-band connectivity during perception of LE syllables, and that (3) enhanced interhemispheric gamma-band connectivity was particularly related to the occurrence of $A V D / A V H$ under ketamine administration.

Currently, there are several models attempting to explain the underlying mechanisms of $\mathrm{AVH}$, including the hypothesis of interhemispheric miscommunication [3, 9]. Several studies using various modalities (DTI, $\mathrm{FMRI}$, and EEG) support the concept that altered connectivity between bilateral auditory areas via the CC is related to the appearance of $\mathrm{AVH}[3,6]$. Concordant with this, it has been shown that abnormal synchrony of oscillatory activity, notably in the gamma-band range, is associated with schizophrenia, leading to neuropsychiatric symptoms such as AVH [4]. Indeed, several studies have indicated that positive symptoms, particularly $\mathrm{AVH}$, are 
related to increased oscillatory gamma-band coupling between circumscribed sensory brain areas [11, 45]. Further support was provided by a recent EEG study showing that the emergence of AVH in patients with schizophrenia was particularly related to stronger interhemispheric gamma-band connectivity between bilateral auditory cortices during LE percepts in a DL task [12]. Although there are several lines of evidence concerning the interhemispheric miscommunication hypothesis on the basis of structural and functional findings, there is not much known about the underlying neurochemical aspects.

Schizophrenia has been associated with disturbed highfrequency GBO [17, 18], which are generated by the interaction of fast-spiking PV interneurons and pyramidal neurons via a feedback loop. While ketamine induces similar effects in healthy humans, such as increasing resting-state gamma-power [25], schizophrenia has been linked to deficient NMDAR functioning [46]. However, somatostatin (SST) interneurons may be more sensitive to deficient NMDAR signaling than PV. As a result, reduced SST activity due to deficient NMDAR signaling would increase PV activity and thus increase GBO. Furthermore, SST inhibition has been associated with hyperconnectivity observed in schizophrenia patients [20]. Therefore, impairments in SST neurons might help to understand that deficient NMDAR functioning results in increased functional connectivity. Thus, altered gamma-band coupling between auditory cortices in $\mathrm{AVH}$, as suggested by the interhemispheric miscommunication hypothesis, might be considered in terms of the NMDAR-hypofunction hypothesis of schizophrenia. NMDAR antagonists are known to induce schizophrenia-like symptoms including $\mathrm{AVH}$ in healthy subjects $[26,47]$ and to induce an E/l imbalance that has been identified as a fundamental contributing factor in the generation of GBO [20]. Moreover, E/l imbalance has also been associated with the appearance of AVH in schizophrenia [23]. In line with this, we found that ketamine modified interhemispheric connectivity in the gamma-band range, and further, increased interhemispheric gamma-band connectivity in healthy subjects with AVD/AVH under ketamine administration.

Although in the ketamine literature ketamine-induced AVH are described only intermittently, one might wonder why half of our participants reported $\mathrm{AVH}$ and/or distortions. One reason could be that asking specifically for AVD/AVH possibly endorses symptoms that participants would not report spontaneously. Additional reasons could be that $A V D / A V H$ have not been investigated consistently in studies with ketamine or auditory alterations were not described in detail [27]. Bowdle et al [47] have noted that plasma concentrations of ketamine correlate with the item "I heard voices or sounds that were not real" of a visual analog rating scale, but this finding was not elaborated on further. Another study assessed hallucinatory psychopathology only with the PANSS P3 item, reflecting hallucinations in any sensory modality and not necessarily auditory hallucinations [28]. However, this study reported detailed case descriptions of healthy subjects with $\mathrm{AVH}$ under ketamine administration and proposed that the occurrence of $\mathrm{AVH}$ is crucially dependent on ketamine dose and environment [28]. The PANSS item P3 is a well-established item of detecting any kind of hallucination, such as visual, olfactory, somatic, and auditory hallucinations. As we were particularly interested in auditory alterations similar to AVH in schizophrenia, we used-in addition to the PANSS-the 5D-ASC questionnaire. The 5D-ASC consists of a detailed subscore detecting auditory alterations (AUA subscore) such as auditory verbal hallucinations and non-verbal hallucinations related to sounds. Furthermore, the 5D-ASC was previously shown to be particularly sensitive to the psychological effects of ketamine [27]. In order to approach AVH in schizophrenia, the present study focussed on items of the AUA subscore related to auditory verbal distortions and hallucinations (AUA-AVD/AVH) and not to any other non-verbal auditory hallucinations. Additionally, as it is known that low doses $(0.003$ $\mathrm{mg} / \mathrm{kg} / \mathrm{min}$ ) of ketamine only elicit mild symptoms such as vigilance reduction, but high doses of ketamine $(0.005 \mathrm{mg} / \mathrm{kg} / \mathrm{min})$ are capable of inducing auditory alterations [29], a high ketamine dose $(0.006 \mathrm{mg} / \mathrm{kg} / \mathrm{min})$ was administered in the current study.

Investigating the interhemispheric miscommunication hypothesis of AVH specifically, we used a DL task. Steinmann et al. [15] demonstrated that interhemispheric auditory connectivity via the $C C$ is especially required during the perception of LE syllables, that interhemispheric transfer is mediated by synchronized GBO, and further, that increased interhemispheric gamma-band connectivity during conscious perception of the LE syllable has been related to AVH in schizophrenia [12].

In accordance with this, we found altered interhemispheric gamma-band connectivity under high-dose ketamine administration during LE percepts, and that increased interhemispheric gamma-band connectivity is related to the appearance of AUAAVD/AVH. One might have expected a modified DL performance during ketamine administration. However, the auditory hallucinatory state has been associated with reduced responsiveness to external auditory stimuli [48].

One limitation of the present study is the relatively low spatial accuracy. However, the use of simultaneous EEG and fMRI has proposed sufficient validity of the LORETA approach in general $[49,50]$. Moreover, connectivity analysis based on EEG rather than fMRI has the advantage of an excellent temporal accuracy measuring direct neuronal activity in the millisecond-range. In addition, the specific strength of the LPS method is that confounding factors such as volume conduction effects are eliminated, and only physiological connectivity information is thought to remain $[40,41]$. The current study shows that the severity of ketamine-induced schizophrenia-like symptoms varies between healthy subjects from no auditory alterations to auditory distortion and $\mathrm{AVH}$. At first glance, this seems to restrain the transfer of the results in healthy volunteers to schizophrenia patients. However, the fact that altered interhemispheric gammaband connectivity has been shown in both, schizophrenia patients with $\mathrm{AVH}$ [12] and healthy volunteers with ketamine-induced AVD/AVH, supports the hypothesis of NMDAR hypofunction as one neurophysiological correlate of $\mathrm{AVH}$ in schizophrenia.

In conclusion, the present study demonstrates for the first time that AVD/AVH induced by NMDAR blockade in healthy subjects are related to increased auditory interhemispheric connectivity in the gamma-band frequency range. Thus, the current study supports the hypothesis that increased gamma-band coupling in AVH might be considered in terms of the NMDAR-hypofunction hypothesis of schizophrenia, and provides further evidence for interhemispheric miscommunication due to $E / I$ imbalance as a correlate of $\mathrm{AVH}$. These results have strong implications for future research about both neurophysiological and the neurochemical mechanisms underlying $\mathrm{AVH}$.

\section{ACKNOWLEDGEMENTS}

We wish to thank the individuals who participated in this study. We would like to thank Sigrid Bozcor for support concerning the statistical analysis. This work was prepared as part of Stephanie Thiebes' dissertation at the University of Hamburg.

\section{FUNDING}

This research was performed within the collaborative research center SFB 936 C6 to Christoph Mulert and supported by the German Research Foundation (DFG). Jürgen Gallinat was supported by DFG SFB 936 C7.

\section{ADDITIONAL INFORMATION}

Supplementary information accompanies this paper at https://doi.org/10.1038/ s41386-018-0014-z.

Conflict of interest The authors declare that they have no conflict of interest 


\section{REFERENCES}

1. Woodruff PW. Auditory hallucinations: Insights and questions from neuroimaging. Cogn Neuropsychiatry. 2004;9:73-91.

2. Hugdahl K. Auditory hallucinations: A review of the ERC "VOICE" project. World J Psychiatry. 2015;5:193-209.

3. Curcic-Blake B, Ford JM, Hubl D, Orlov ND, Sommer IE, Waters F, et al. Interaction of language, auditory and memory brain networks in auditory verbal hallucinations. Prog Neurobiol. 2017;148:1-20.

4. Uhlhaas PJ, Singer W. High-frequency oscillations and the neurobiology of schizophrenia. Dialog Clin Neurosci. 2013;15:301-13.

5. Singer W. Neuronal synchrony: a versatile code for the definition of relations? Neuron. 1999;24:49-65. 111-25

6. Steinmann S, Leicht G, Mulert C. Interhemispheric auditory connectivity: structure and function related to auditory verbal hallucinations. Front Human Neurosci. 2014;8:55.

7. Hubl D, Koenig T, Strik W, Federspiel A, Kreis R, Boesch C, et al. Pathways that make voices: white matter changes in auditory hallucinations. Arch Gen Psychiatry. 2004;61:658-68.

8. Leroux $\mathrm{E}$, Delcroix N, Dollfus $\mathrm{S}$. Abnormalities of language pathways in schizophrenia patients with and without a lifetime history of auditory verbal hallucinations: A DTI-based tractography study. World J Biol Psychiatry. 2017;18:528-38.

9. Mulert C, Kirsch V, Whitford TJ, Alvarado J, Pelavin P, McCarley RW, et al. Hearing voices: a role of interhemispheric auditory connectivity? World J Biol Psychiatry. 2012;13:153-8.

10. Gavrilescu M, Rossell S, Stuart GW, Shea TL, Innes-Brown H, Henshall K, et al. Reduced connectivity of the auditory cortex in patients with auditory hallucinations: a resting state functional magnetic resonance imaging study. Psychol Med. 2010;40:1149-58.

11. Mulert C, Kirsch V, Pascual-Marqui R, McCarley RW, Spencer KM. Long-range synchrony of gamma oscillations and auditory hallucination symptoms in schizophrenia. Int J Psychophysiol. 2011;79:55-63.

12. Steinmann S, Leicht G, Andreou C, Polomac N, Mulert C. Auditory verbal hallucinations related to altered long-range synchrony of gamma-band oscillations. Sci Rep. 2017;7:8401.

13. Brancucci A, Babiloni C, Babiloni F, Galderisi S, Mucci A, Tecchio F, et al. Inhibition of auditory cortical responses to ipsilateral stimuli during dichotic listening: evidence from magnetoencephalography. Eur J Neurosci. 2004;19:2329-36.

14. Kimura D. Functional asymmetry of the brain in dichotic listening. Cortex. 1967;3:163-8.

15. Steinmann S, Leicht G, Ertl M, Andreou C, Polomac N, Westerhausen R, et al. Conscious auditory perception related to long-range synchrony of gamma oscillations. Neurolmage. 2014a;100:435-43.

16. Traub RD, Whittington MA, Stanford IM, Jefferys JG. A mechanism for generation of long-range synchronous fast oscillations in the cortex. Nature. 1996;383:621-4.

17. Lee KH, Williams LM, Breakspear M, Gordon E. Synchronous gamma activity: a review and contribution to an integrative neuroscience model of schizophrenia. Brain Res Brain Res Rev. 2003;41:57-78.

18. Uhlhaas PJ, Singer W. Abnormal neural oscillations and synchrony in schizophrenia. Nat Rev Neurosci. 2010;11:100-13.

19. Yizhar O, Fenno LE, Prigge M, Schneider F, Davidson TJ, O'Shea DJ, et al. Neocortical excitation/inhibition balance in information processing and social dysfunction. Nature. 2011:477:171-8.

20. Krystal JH, Anticevic A, Yang GJ, Dragoi G, Driesen NR, Wang XJ, et al. Impaired tuning of neural ensembles and the pathophysiology of schizophrenia: a translational and computational neuroscience perspective. Biol Psychiatry. 2017;81:874-85.

21. Tatti R, Haley MS, Swanson OK, Tselha T, Maffei A. Neurophysiology and regulation of the balance between excitation and inhibition in neocortical circuits. Biol Psychiatry. 2017;81:821-31.

22. Foss-Feig JH, Adkinson BD, Ji JL, Yang G, Srihari VH, McPartland JC, et al. Searching for cross-diagnostic convergence: neural mechanisms governing excitation and inhibition balance in schizophrenia and autism spectrum disorders. Biol Psychiatry. 2017;81:848-61.

23. Jardri R, Hugdahl K, Hughes M, Brunelin J, Waters F, Alderson-Day B, et al. Are hallucinations due to an imbalance between excitatory and inhibitory influences on the brain? Schizophr Bull. 2016;42:1124-34.

24. Gandal MJ, Sisti J, Klook K, Ortinski PI, Leitman V, Liang Y, et al. GABAB-mediated rescue of altered excitatory-inhibitory balance, gamma synchrony and behavioral deficits following constitutive NMDAR-hypofunction. Transl Psychiatry. 2012;2:e142.

25. Rivolta D, Heidegger T, Scheller B, Sauer A, Schaum M, Birkner K, et al. Ketamine dysregulates the amplitude and connectivity of high-frequency oscillations in cortical-subcortical networks in humans: evidence from resting-state magnetoencephalography-recordings. Schizophr Bull. 2015;41:1105-14.

26. Lahti AC, Weiler MA, Tamara Michaelidis BA, Parwani A, Tamminga CA. Effects of ketamine in normal and schizophrenic volunteers. Neuropsychopharmacology. 2001;25:455-67.

27. Musso F, Brinkmeyer J, Ecker D, London MK, Thieme G, Warbrick T, et al. Ketamine effects on brain function-simultaneous $\mathrm{FMRI} / \mathrm{EEG}$ during a visual oddball task. Neuroimage. 2011;58:508-25.

28. Powers AR 3rd, Gancsos MG, Finn ES, Morgan PT, Corlett PR. Ketamine-induced hallucinations. Psychopathology. 2015;48:376-85.

29. Passie T, Karst M, Borsutzky M, Wiese B, Emrich HM, Schneider U. Effects of different subanaesthetic doses of (S)-ketamine on psychopathology and binocular depth inversion in man. J Psychopharmacol. 2003;17:51-56.

30. Thiebes S, Leicht G, Curic S, Steinmann S, Polomac N, Andreou C, et al. Glutamatergic deficit and schizophrenia-like negative symptoms: new evidence from ketamine-induced mismatch negativity alterations in healthy male humans. Psychiatry Neurosci. 2017;42:273-83.

31. Oldfield RC. The assessment and analysis of handedness: the Edinburgh inventory. Neuropsychologia. 1971;9:97-113.

32. Sheehan DV, Lecrubier $\mathrm{Y}$, Sheehan $\mathrm{KH}$, Amorim $\mathrm{P}$, Janavs J, Weiller $\mathrm{E}$, et al. The Mini-International Neuropsychiatric Interview (M.I.N.I.): the development and validation of a structured diagnostic psychiatric interview for DSM-IV and ICD-10. J Clin Psychiatry. 1998;59 Suppl 20:22-33.

33. Raine A. The SPQ: a scale for the assessment of schizotypal personality based on DSM-III-R criteria. Schizophr Bull. 1991;17:555-64.

34. Herzfeld H. WST-Wortschatztest diagnostica. Weinheim: Beltz Test GmbH; 1994

35. Kay SR, Fiszbein A, Opler LA. The positive and negative syndrome scale (PANSS) for schizophrenia. Schizophr Bull. 1987;13:261-76.

36. van der Gaag M, Hoffman T, Remijsen M, Hijman R, de Haan L, van Meijel B, et al The five-factor model of the Positive and Negative Syndrome Scale II: a ten-fold cross-validation of a revised model. Schizophr Res. 2006;85:280-7.

37. Dittrich A. The standardized psychometric assessment of altered states of consciousness (ASCs) in humans. Pharmacopsychiatry. 1998;31 Suppl 2:80-84.

38. Pascual-Marqui RD (2007). Discrete, 3D distributed, linear imaging methods of electric neuronal activity. Part 1: Exact, zero error localization, (arXiv:0710.3341 [math-ph], 17 October, http://arxiv.org/abs/0710.3341). 2007.

39. Pascual-Marqui RD. Theory of the EEG inverse problem, Ch 5. Boston, MA: Artech House; 2009. P. .

40. Pascual-Marqui RD (2007). Instantaneous and lagged measurements of linear and nonlinear dependence between groups of multivariate time series: frequency decomposition (arXiv: 0711.1455 [stat.ME], 2007-November-09, http://arxiv.org/ abs/0711.1455). 2007.

41. Pascual-Marqui RD, Lehmann D, Koukkou M, Kochi K, Anderer P, Saletu B, et al. Assessing interactions in the brain with exact low-resolution electromagnetic tomography. Philos Trans A Math Phys Eng Sci. 2011;369:3768-84.

42. Gueorguieva R, Krystal JH. Move over ANOVA: progress in analyzing repeatedmeasures data and its reflection in papers published in the Archives of General Psychiatry. Arch Gen Psychiatry. 2004;61:310-7.

43. Andreou C, Kleinert J, Steinmann S, Fuger U, Leicht G, Mulert C. Oscillatory responses to reward processing in borderline personality disorder. World $\mathrm{J}$ Biol Psychiatry. 2015;16:575-86.

44. Bachman P, Kim J, Yee CM, Therman S, Manninen M, Lonnqvist J, et al. Abnormally high EEG alpha synchrony during working memory maintenance in twins discordant for schizophrenia. Schizophr Res. 2008;103:293-7.

45. Spencer KM, Nestor PG, Niznikiewicz MA, Salisbury DF, Shenton ME, McCarley RW. Abnormal neural synchrony in schizophrenia. J Neurosci. 2003;23:7407-11.

46. Moghaddam B. Bringing order to the glutamate chaos in schizophrenia. Neuron. 2004;40:881-4.

47. Bowdle TA, Radant AD, Cowley DS, Kharasch ED, Strassman RJ, Roy-Byrne PP. Psychedelic effects of ketamine in healthy volunteers: relationship to steady-state plasma concentrations. Anesthesiology. 1998;88:82-88.

48. Hubl D, Koenig T, Strik WK, Garcia LM, Dierks T. Competition for neuronal resources: how hallucinations make themselves heard. $\mathrm{Br} J$ Psychiatry. 2007;190:57-62.

49. Mulert C, Jager L, Propp S, Karch S, Stormann S, Pogarell O, et al. Sound level dependence of the primary auditory cortex: Simultaneous measurement with 61channel EEG and fMRI. Neuroimage. 2005;28:49-58.

50. Mulert $C$, Jager L, Schmitt R, Bussfeld $P$, Pogarell $O$, Moller HJ, et al. Integration of fMRI and simultaneous EEG: towards a comprehensive understanding of localization and time-course of brain activity in target detection. Neuroimage. 2004;22:83-94. 